• 研究报告・

\title{
铁角蕨科的多倍化与物种多样性形成
}

\author{
常艳芬 ${ }^{*}$ \\ (中国科学院西双版纳热带植物园, 云南㔜腊 666303)
}

\begin{abstract}
摘要: 铁角蒴科植物约 800 种, 广布世界各地, 主产热带和亚热带地区。本文统计了铁角藓科188种植物的细胞学 资料, 发现 167 种植物具有多倍化现象, 占总种数的 $88.8 \%$, 表明该类群植物中普遍存在多倍化事件。具有多倍化 现象的物种中, 90 种只有 1 种细胞型, 占总种数的 $47.9 \%$; 77 种具有种内多倍性, 即种下存在多个细胞型组合, 占总 种数的 $41.0 \%$ 。多倍体细胞倍性极其丰富, 有三倍体、四倍体、六倍体、八倍体、十倍体、十二倍体以及十六倍 体。本文还对铁角蒴科多倍化现象与其物种多样性形成的关系进行了讨论, 同时对铁角硕科多倍化研究中存在的 问题进行了探讨。
\end{abstract}

关键词：䕬类植物; 多倍化; 物种多样性; 网状进化; 无融合生殖

\section{Polyploidy and the formation of species diversity in Aspleniaceae}

\author{
Yanfen Chang* \\ Xishuangbanna Tropical Botanical Garden, Chinese Academy of Sciences, Mengla, Yunnan 666303
}

\begin{abstract}
Ferns are considered to have the highest frequency of polyploidy in plants. Based on the published cytological data of 188 species, we analyzed the relationship between polyploidy and the formation of species diversity in the fern family Aspleniaceae, which comprises approximately 800 species. The results show that polyploids, including triploids, tetraploids, hexaploids, octoploids, decaploids, dodecaploids and hexadecaploids, have been documented in the family. Of the 188 Aspleniaceae species with cytological data, 88.8\% exhibit polyploidy, $41.0 \%$ show intraspecific polyploidy and $47.9 \%$ are the result of polyploid speciation. In addition, the diverse ploidy levels suggest that these species have a complex evolutionary history and their taxonomic problems require further study. The perplexity and future directions of study of Aspleniaceae were also discussed.
\end{abstract}

Key words: Pteridophytes; polyploid; species diversity; reticulation; apomixes

多倍化指产生多倍体的过程，通常包括同源多 倍体和异源多倍体两种类型(Jackson, 1976; Grant, 1981; 洪德元, 1990)。多倍化为植物的物种进化提 供了一个快速通道, 是植物最重要的遗传变异之一, 在植物的物种多样性形成中具有重要的意义(Grant, 1981; Rieseberg \& Willis, 2007)。植物通过杂交产生 的后代由于具有不同的基因组, 其染色体同源性较 低, 在减数分裂时不能形成正常的配子体, 往往导 致杂交后代不育; 即使后代可育, 也会由于染色体 之间的同源性低, 表达的不协调以及核质互作等原 因导致杂种衰退。然而, 如果杂交后代发生染色体
加倍, 则其染色体在减数分裂时就可以正常配对, 不仅可以避免杂种衰退，而且亲本基因在形成合子 后，常常会发生基因重组或重排，可以丰富物种的 基因库并加速基因的突变频率，使杂种后代形成杂 种优势, 具有比亲本更强的适应性。因此, 杂交后 的多倍化过程是非常重要的物种形成机制, 是所谓 量子物种形成(quantum speciation)、同域物种形成 (sympatric speciation)以及生态物种形成(ecological speciation)的重要原因(Rieseberg \& Willis, 2007)。

痥类植物的多倍体比例在植物界是最高的(王 任翔等, 2007; Wood et al, 2009)。其中, 铁角硕科就

收稿日期: 2017-04-13; 接受日期: 2017-06-28

基金项目: 国家自然科学基金(31500171)和生物标本馆经典分类学青年人才项目(ZSBR-008)

* 通讯作者 Author for correspondence. E-mail: cyf@xtbg.org.cn 
因有着丰富的多倍化现象而成为研究蒴类植物物 种形成和进化机制的模式类群(Wagner, 1954; Lovis, 1977; Reichstein, 1981; Werth et al, 1985)。该类群物 种丰富, 广布世界各地, 主产热带和亚热带地区, 由700余种的铁角偋属(Asplenium)和约30种的膜叶 铁角偋属(Hymenasplenium)组成, 共约800种 (吴兆 洪, 1999; Lin \& Viane, 2012; 张宪春, 2012; 张宪春 等, 2013)。该类群中多倍体倍性最高达到了16倍, 是烣类植物细胞倍性水平最高的类群(Braithwaite, 1986)。迄今为止, 我们对多倍化与铁角蒴科中物种 多样性形成的关系还知之甚少。

本文介绍了铁角偋科植物中多倍化的研究现 状, 并对该类群植物中多倍化与物种多样性形成的 关系进行了探讨, 同时对铁角蒝科植物多倍化研究 中存在的问题及未来的研究方向进行了讨论, 旨在 进一步推动铁角偋科植物的物种起源与分化及其 演化规律的研究。

\section{1 材料与方法}

通过收集和整理Tropicos (http://www.tropicos. org)数据库中记载的铁角偋科植物的染色体数目信 息，以及查阅相关的文献及著作(Kato et al，1992; Cheng \& Zhang, 2010; Lin \& Viane, 2012; Liu et al, 2012), 得到铁角䓲科植物中已有报道的细胞学数 据。为便于分析, 本文将种下分类群的细胞学数据 处理到种级分类群的数据。

\section{2 结果}

\section{1 铁角薮科植物的多倍化情况}

据整理, 铁角蒝科中共有 188 种植物有细胞学 数据(附录1)。其中 167 种具有多倍化现象, 占总种数 的 $88.8 \%$; 21种为二倍体种，占总种数的 $11.2 \%$ 。多 倍化的倍性多样, 有三倍体、四倍体、六倍体、八 倍体、十倍体、十二倍体以及十六倍体。167种具 有多倍化的物种中, 90 种具单一倍性(种下仅有单一 细胞型), 占总种数的 $47.9 \%$, 其中四倍体种最多, 有67种; 三倍体种有2种; 六倍体种有11种; 八倍体 种有 9 种; 十倍体种有 1 种。

\section{2 种内多倍性}

铁角偋科具有多倍化现象的 167 种中, 77 种具 有种内多倍性, 即种下存在多个细胞型, 占总种数 的 $41.0 \%$ 。其中, 具有 2 种细胞型的有 57 种, 最普遍
的是二倍体和四倍体的组合, 有33种; 其他具有 2 种细胞型组合的还有二倍体和三倍体(或八倍体), 三倍体和四倍体(或六倍体), 四倍体和六倍体(或八 倍体)以及八倍体和六倍体(或十六倍体)等的组合。 具有 3 种细胞型的有 16 种, 分别是二倍体、三倍体和 四倍体, 二倍体、四倍体和六倍体(或八倍体和十二 倍体), 以及四倍体、六倍体(或八倍体和十二倍体)。 具有 4 种细胞型的有 4 种, 其中, 药茨 (Asplenium ceterach) 和铁角蕨(A. trichomanes)具有二倍体、三 倍体、四倍体及六倍体, 倒挂铁角蕨(A. normale)中 含有二倍体、四倍体、六倍体及八倍体, A. aethiopicum中含有四倍体、八倍体、十二倍体及十六倍体。

\section{3 无融合生殖及非整倍体}

铁角葓科中 43 种植物有繁殖方式的记录, 其中 10 种存在无融合生殖, 占比为 $23.3 \%$, 其余均行有 性生殖。然而, 在膜叶铁角蒝属中, 18种植物中就有 5 种具有无融合生殖方式, 占比高达 $28 \%$ 。在这些无 融合生殖的类群中, 三倍体是最多的。例如膜叶铁 角偋属中的三倍体均为行无融合生殖的类群, 如齿 果膜叶铁角偋(Hymenasplenium cheilosorum)、东亚

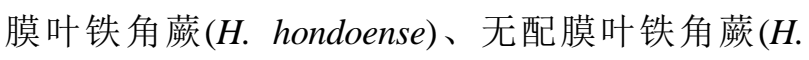
apogamum) 和荫湿膜叶铁角䓲 (H. obliquissimum); 铁角硕属的A. curtissii、A. dentatum和A. monanthes 的三倍体也行无融合生殖。

铁角蒝属中, 大部分物种的染色体基数为 $\mathrm{x}=$ 36, 而在Asplenium centrafricanum、A. diplazisorum、 A. loxoscaphoides、A. rutifolium、A. sertularioides、 以及 $A$. theciferum中的染色体数目均为 $\mathrm{x}=35$ 的整 倍数, 染色体基数为 $\mathrm{x}=36$ 的非整倍体变异。在膜叶 铁角䓲属中, 大多数物种的染色体基数为 $\mathrm{x}=39$, 而在Hymenasplenium subnormale中出现了例外, 染 色体基数为 $\mathrm{x}=38$ 。

\section{3 讨论}

\section{1 铁角蕨科的多倍化与物种多样性形成}

多倍化是植物物种多样性形成的一个重要过 程(Rieseberg \& Willis, 2007)。种子植物中多倍体种 的比例是 $15 \%$, 䓲类植物中多倍体种的比例高达 $31 \%$ (Wood et al, 2009)。而在有细胞学数据记录的 188种铁角蒝科植物中, 90 种为单一倍性的多倍体 种, 占总种数的 $47.9 \%$ 。由此可见, 多倍化成种在铁 角蕨科植物的物种多样性形成中具有重要作用。 
铁角葓科中大量的多倍体种的形成可能与其 地理分布有关。Manton (1959)对蕨类植物多倍体种 分布的研究结果显示, 欧洲温带地区的多倍体种的 比例均不到 $50 \%$, 而非洲热带和美洲热带地区分布 的多倍体种比例均超过60\%。Wood等(2009)对美洲 热带地区木本植物多倍体种分布的研究也得到相 似的结果。铁角偋科植物主要分布于热带地区, 该 地区生物多样性异常丰富, 物种可能产生更多的变 异来提供重组机会, 从而有助于形成更多的物种 (Haufler et al, 2000; Haufler, 2002; Hendry, 2009)。另 外, 有研究认为, 极端生境条件也是多倍化产生的 重要因素(洪德元, 1990)。铁角硕科植物广泛分布在 世界各地, 多为石生或附生, 其普遍的多倍化现象 也可能与其特殊生境有关。

\section{2 铁角烣科的种内多倍化与物种多样性形成}

铁角蕨科中 $41.0 \%$ 的物种中存在种内多倍性, 这些物种不是单型种, 而是复杂的复合体种, 种间 除了具有不同的细胞倍性, 还常常在形态特征、生 境、生殖方式等方面也存在变异。另外, 这些复合 体种中的多倍化常常与杂交共同作用, 形成的杂种 由于具有两个亲本的基因组, 形态特征常介于两个 亲本之间, 形成中间过渡形态, 使种与种之间形成 复杂的网状进化关系, 种间界限变得模糊不清。研 究这些复合体种的种间关系及物种分类异常困难。 目前为止, 只有少量的复合体种得到了比较详细的 研究。

例如, 北美分布的Appalachian铁角硕复合体是 铁角硕科中著名的研究网状进化的例子。该复合体 种涉及11个分类群, 由3个二倍体祖先种A. montanum、A. rhizophyllum和A. platyneuron相互杂交, 随 后发生多倍化并回交, 最终形成二倍体杂种 $A$. ebenoides, 以及异源四倍体种A. gravesii、A. pinnatifidum、A. ebenoides、A. bradleyi和三倍体种 $A$. trudellii、A. bradleyi、A. kentuckiense。这些类群相 互之间又会进行新一轮的杂交, 最后形成错综复杂 的网状进化关系(Wagner, 1954; Werth et al, 1985)。 铁角硕复合体(A. trichomanes complex)由祖先二倍 体的原亚种铁角蕨(A. t. subsp. trichomanes)和喜钙 亚种(A. t. subsp. inexpectans)杂交或自行加倍, 衍生 出异源四倍体亚种A. t. subsp. quadrivalen、A. $t$. subsp. hastatum, 以及同源四倍体粗轴亚种 (A. $t$. subsp. pachyrachis); 这些四倍体种和另一个四倍体
A. t. subsp. coriaceifolium进行杂交组合再产生四倍 体A. t. nothosubsp. lovisianum、A. t. nothosubsp. moravicum和A. t. nothosubsp. staufferi (Lovis, 1964; Bennert \& Fischer, 1993; 侯金和王中仁, 2000; Ekrt \& Stech, 2008)。目前的分子证据提示东亚分布的三 翅铁角硕 (A. tripteropus) 和产太平洋岛屿的 A. anceps 可能也属于铁角硕复合体, 但它们与铁角䓲 复合体其他类群之间的演化关系还需要进一步的 研究(Schneider et al, 2004, 2013)。分布于亚洲的华 中铁角偋复合体(A. sarelii complex)由 3 个基本的二 倍体祖先种, 即华中铁角蒝(A. sarelii)、细茎铁角蒝 (A. tenuicaule)和泸山铁角硕(A. lushanense)经过自 身染色体加倍, 形成同源四倍体北京铁角硕(A. pekinense)、变异铁角蕨(A. varians) 和云南铁角蕨(A. yunnanense), 这些类群间进一步杂交衍生出新的分 类群, 最终使华中铁角蒝复合体的成员达到 13 个之 多(Wang et al, 2003)。

另外, 巢葓(Aspelnium nidus)也可能是一个复 杂的复合体种, 该复合种内也存在复杂的杂交及多 倍化过程, 并存在至少 6 个隐存种, 因此导致对该 类群进行分类学研究异常困难(Yatabe et al, 2001, 2009; Yatabe \& Murakami, 2003); 药䔸复合体(Aspelnium ceterach complex)的多倍体类群A. aureum、

A. hybridumhas、A. lolegnamense、A. punjabense以 及A. octoploideum等为异源多倍体, 它们的起源也 与网状进化紧密相连(Pinter et al, 2002; van den Heede et al, 2003); 新西兰产澳大利亚铁角䓲类群 (Austral group)的8个八倍体类群中至少有 7 个来源 于7个四倍体类群的杂交组合(Shepherd et al, 2008); A. monanthes complex复合体和倒挂铁角硕复合体 (A. normale complex)中也存在着复杂的网状进化 (Dyer et al, 2012; Chang et al, 2013)。这些复合体种 的物种分类及网状进化关系还需要进一步研究。

相对于铁角硕科内广泛存在的多倍化和网状 进化事件, 上述研究揭示的可能还只是冰山一角。 研究网状进化关系的关键是阐明复合体种内多倍 体类群的起源, 然而, 由于选择压力、遗传漂变等 因素的影响, 多倍体种与祖先种的性状常常有明显 间断, 甚至有的祖先种已灭绝, 导致研究异常困 难。由于铁角偋科中存在大量的杂交和多倍化事件, 因此, 只有阐明了多倍化在铁角葓科的分布和复杂 程度及其对该类群系统演化的影响, 才能阐明其演 
化历史, 并最终得到一个可以被广泛接受的铁角蕨 科系统。

\section{3 铁角蕨科的非整倍性及无融合生殖与物种多 样性形成}

染色体的非整倍体变异是植物进化的一个重 要过程(Bellefroid et al, 2010)。膜叶铁角硕属中少数 物种的染色体基数为 $\mathrm{x}=38$ (Mitui et al, 1989; Cheng \& Murakami, 1998), 可能是由染色体基数为 $\mathrm{x}=39$ 的祖先核型演化而来, 而铁角蒝属中少数物 种的染色体基数为 $x=35$, 则可能是由染色体基数 为 $x=36$ 的祖先核型演化而来(Bellefroid et al, 2010), 而且非整倍体变异可能在铁角葓科中发生了多次 起源, 可能是促进该类群植物物种分化的原因之一 (Bellefroid et al, 2010)。

蒝类植物的无融合生殖是指未经受精的配子 体直接发育成狍子体(洪德元, 1990; Grusz, 2016)。 据统计, 偋类植物中约 $10 \%$ 的物种存在无融合生殖, 该比例远远高于种子植物的不到 $1 \%$ (van den Heede et al, 2003; Liu et al, 2012)。而铁角偋科中的无融合 生殖类群所占比例更高。因此, 无融合生殖方式可 能在铁角偋科植物的进化过程中发挥着巨大的作 用。而且, 在铁角偋科无融合生殖的类群中, 三倍 体行无融合生殖是最多的。所以, 有必要对三倍体 的物种形成与无融合生殖的关系进行深入的研究。 另外, 目前对铁角偋科中一些具有无融合生殖的类 群研究发现, 大多无融合生殖类群虽然是行无性生 殖却具有较高的遗传变异, 它们常常与其他无融合 生殖类群或有性生殖类群形成复杂的网状进化关 系, 或形成复杂的复合体种, 例如 A. resiliens、A. palmeri、A. hallbergii和A. monanthes complex (Ebihara et al, 2005; Grusz et al, 2009; Dyer et al, 2012; Hori et al, 2014)。因此, 无融合生殖可能与铁角蒝科 的物种多样性形成具有密切的关系。

\section{4 铁角烣科多倍化研究中存在的问题}

\subsection{1 研究不够充分}

有研究指出, 热带地区多样性异常丰富, 物种 可能产生更多的变异来提供重组机会(Haufler et al, 2000; Haufler, 2002; Hendry, 2009), 因此, 分布于 热带地区的铁角偋科植物的多倍体数目可能会更 多, 其多倍化程度可能比我们想象的要复杂得多。 然而, 目前对铁角蒝科的细胞学和系统学研究大多 基于欧洲和北美的类群。因此, 要详细了解铁角烣
科的多倍化情况, 还需要进一步扩大对该类群的研 究范围。

\subsection{2 形态分类和物种鉴定困难}

铁角硕科中丰富的细胞倍性暗示着该类群可 能具有复杂的进化历史, 复合体种中可能还存在隐 存种(Yatabe et al, 2001, 2009; Yatabe \& Murakami, 2009; Chang et al, 2013), 这些类群的物种分类还需 要进一步的澄清。目前对铁角蒴科植物的物种分类 研究主要基于形态学。然而, 大量的杂交和多倍化 事件导致该类群植物形态变异较大, 性状重叠, 而 且形态性状经历了多次起源(Murakami et al, 1999; Schneider et al, 2004; 顾钰峰等, 2014)。只靠形态特 征对铁角蒴科进行物种分类研究容易产生分类学 上的混乱。因此, 除了形态特征外, 研究该类群的物 种分类还需要综合考虑其他方面的证据，例如分类 群的细胞学特征、遗传学特征和地理分布格局等。

\subsection{3 网状进化研究困难}

多倍化及网状进化在铁角蒝科的进化中起到 了至关重要的作用。只有充分了解铁角偋科中的多 倍化事件, 并深入研究其物种间的网状进化关系, 才能真正认识该类群的物种分类及物种间复杂的 演化关系。然而, 目前对铁角偋科系统发育的研究 大多基于叶绿体基因组的DNA序列(Schneider et al, 2013)。叶绿体基因组进化速率缓慢, 很难用于解决 种下水平分类群间的系统发育关系及研究最近发 生的进化事件(Sang \& Zhong, 2000; Brysting et al, 2007，2011）。而核基因不仅进化速率快，且网状进 化容易在单亲遗传的叶绿体基因片段和双亲遗传 的核基因片段上以基因树冲突的形式表现出来(邹 新慧和葛颂, 2008)。因此, 要正确理解铁角偋科的 多倍化和网状进化，必须引入具有双亲遗传特性的 核基因分子序列。近来有研究显示，单(低)拷贝核基 因片段在揭示铁角䓲科网状进化事件时起到了关 键作用(Dyer et al, 2012; Chang et al, 2013; Schneider et $\mathrm{al}$, 2013), 特别是通过多个单(低)拷贝核基因序 列的联合使用，可以有效区分直系同源或旁系同源 以及杂合态等位基因或祖先多态性等因素的影响, 从而在探讨网状进化关系上十分有效(Schneider et al, 2013)。

\section{参考文献}

Bellefroid E, Rambe K, Leroux O, Viane R (2010) The base 
number of 'loxoscaphoid' Asplenium species and its implication for cytoevolution in Aspleniaceae. Annals of Botany, 106, 157-171.

Bennert HW, Fischer G (1993) Biosystematics and evolution of the Asplenium trichomanes complex. Webbia, 48, 743-760.

Braithwaite AF (1986) The Asplenium aethiopicum complex in South Africa. Botanical Journal of the Linnean Society, 93, 343-378.

Brysting AK, Mathiesen C, Marcussen T (2011) Challenges in polyploid phylogenetic reconstruction: a case story from the arctic-alpine Cerastium alpinum complex. Taxon, 60, 333347.

Brysting AK, Oxelman B, Huber KT, Moulton V, Brochmann C (2007) Untangling complex histories of genome mergings in high polyploids. Systematic Biology, 56, 467-476.

Chang YF, Li J, Lu SG, Schneider H (2013) Species diversity and reticulate evolution in the Asplenium normale complex (Aspleniaceae) in China and adjacent areas. Taxon, 62, 673687.

Cheng X, Murakami N (1998) Cytotaxonomic study of genus Hymenasplenium (Aspleniaceae) in Xishuangbanna, southwestern China. Journal of Plant Research, 111, 495-500.

Cheng X, Zhang SZ (2010) Index to chromosome numbers of Chinese Pteridophyta (1969-2009). Journal of Fairylake Botanic Garden, 9, 1-58.

Dyer RJ, Savolainen V, Schneider H (2012) Apomixis and reticulate evolution in the Asplenium monanthes fern complex. Annals of Botany, 110, 1515-1529.

Ebihara A, Ishikawa H, Matsumoto S, Lin SU, Iwatsuki K, Takamiya M, Watano Y, Ito M (2005) Nuclear DNA, chloroplast DNA, and ploidy analysis clarified biological complexity of the Vandenboschia radicans complex (Hymenophyllaceae) in Japan and adjacent areas. American Journal of Botany, 92, 1535-1547.

Ekrt L, Stech M (2008) A morphometric study and revision of the Asplenium trichomanes group in the Czech Republic. Preslia, 80, 325-347.

Grant V (1981) Plant Speciation, pp. 273-317. Columbia University Press, New York.

Grusz AL (2016) A current perspective on apomixis in ferns. Journal of Systematics and Evolution, 54, 656-665.

Grusz AL, Windham MD, Pryer KM (2009) Deciphering the origins of apomictic polyploids in the Cheilanthes yavapensis complex (Pteridaceae). American Journal of Botany, 96, 1636-1645.

Gu YF, Wei HJ, Wei R, Dai XL, Yan YH (2014) Diplazium × kidoi Sa. Kurata, a newly recorded species of Diplazium (Athyriaceae) from China. Plant Science Journal, 32, 336-339. (in Chinese with English abstract) [顾钰峰, 韦宏 金, 卫然, 戴锡玲, 严岳鸿 (2014) 中国双盖䓲属一新记 录种—Diplazium $\times$ kidoi Sa. Kurata. 植物科学学报, 32, 336-339.]

Haufler CH (2002) Homospory 2002: an odyssey of progress in pteridophyte genetics and evolutionary biology. BioScience, 52, 1081-1093.

Haufler CH, Hooper EA, Thierrien JP (2000) Modes and mechanisms of speciation in pteridophytes; implications of contrasting patterns in ferns representing temperate and tropical habitats. Plant Species Biology, 15, 223-236.

Hendry AP (2009) Evolutionary biology: speciation. Nature, 458, 162-164.

Hong DY (1990) Plant Cytotaxonomy. Science Press, Beijing. (in Chinese) [洪德元 (1990) 植物细胞分类学. 科学出版 社, 北京.]

Hori K, Tono A, Fujimoto K, Kato J, Ebihara A, Watano Y, Murakami N (2014) Reticulate evolution in the apogamous Dryopteris varia complex (Dryopteridaceae, subg. Erythrovariae, sect. Variae) and its related sexual species in Japan. Journal of Plant Research, 127, 661-684.

Hou X, Wang ZR (2000) A subspecific taxonomic study on Asplenium trichomanes L. from China. Acta Phytotaxonomica Sinica, 38, 242-255. (in Chinese with English abstract) [侯金金, 王中仁 (2000) 中国铁角蕨的种下分类学 研究. 植物分类学报, 38, 242-255.]

Jackson RC (1976) Evolution and systematic significance of polypolidy. Annual Review of Ecology and Systematics, 7, 209.

Kato M, Nakato N, Cheng X, Iwatsuki K (1992) Cytotaxonomic study of ferns of Yunnan, southwestern China. Journal of Plant Research, 105, 105-124.

Lin YX, Viane R (2012) Aspleniaceae. In: Flora of China (ed. Editorial Committee of Flora of China). Science Press, Beijing \& Missouri Botanical Garden Press, St. Louis.

Liu HM, Dyer RJ, Guo ZY, Meng Z, Li JH, Schneider H (2012) The evolutionary dynamics of apomixis in ferns: a case study from polystichoid ferns. Journal of Botany, 2012, http://dx.doi.org/10.1155/2012/510478.

Lovis JD (1964) The taxonomy of Asplenium trichomanes in Europe. British Fern Gazette, 9, 147-160.

Lovis JD (1977) Evolutionary patterns and processes in ferns. Advances in Botanical Research, 4, 229-415.

Manton I (1959) Cytological information on the ferns of West Tropical Africa. In: The Ferns and Fern Allies of West Tropical Africa (ed. Alston AHG). Grown Agents, London.

Mitui K, Murakami N, Iwatsuki K (1989) Chromosomes and systematics of Asplenium sect. Hymenasplenium (Aspleniaceae). American Journal of Botany, 76, 1689-1697.

Murakami N, Nogami S, Watanabe M, Iwatsuki K (1999) Phylogeny of Aspleniaceae inferred from $r b c \mathrm{~L}$ nucleotide sequences. American Fern Journal, 89, 232-243.

Nyhus GC (1987) The subspecies of Asplenium trichomanes in Norway. Blyttia, 45, 12-24.

Perrie LR, Brownsey PJ (2005) Insights into the biogeography and polyploid evolution of New Zealand Asplenium from chloroplast DNA sequence data. American Fern Journal, 95, $1-21$.

Pinter I, Bakker F, Barrett JA, Cox C, Gibby M, Henderson S, Morgan-Richards M, Rumsey F, Russell S, Trewick S, Schneider H, Vogel J (2002) Phylogenetic and biosystematic relationships in four highly disjunct polyploidy complexes in the subgenera Ceterach and Phyllitis in Asplenium (Aspleniaceae). Organisms, Diversity and Evolution, 2, 299- 
311.

Rasbach H, Rasbach K, Reichstein T, Bennert HW (1990) Asplenium trichomanes subsp. coriaceifolium, a new subspecies and two new intraspecific hybrids of the A. trichomanes complex (Aspleniaceae, Pteridophyta). I. Nomenclature and typification. Willdenowia, 19, 471-474.

Rasbach H, Rasbach K, Reichstein T, Bennert HW (1991) Asplenium trichomanes subsp. coriaceifolium, a new subspecies and two new intraspecific hybrids of the A. trichomanes complex (Aspleniaceae, Pteridophyta). II. Description and illustrations. With an appendix on pairing behaviour of chromosomes in fern hybrids. Willdenowia, 21, 239-261.

Reichstein T (1981) Hybrids in European Aspleniaceae (Pteridophyta). Botanica Helvetica, 91, 89-139.

Rieseberg LH, Willis JH (2007) Plant speciation. Science, 317, 910-914.

Sang T, Zhong Y (2000) Testing hybridization hypotheses based on incongruent gene trees. Systematic Biology, 49, 422-434.

Schneider H, Navarro-gomez A, Russell SJ, Ansell S, Grundmann M, Vogel J (2013) Exploring the utility of three nuclear regions to reconstruct reticulate evolution in the fern genus Asplenium. Journal of Systematics and Evolution, 51, 142-153.

Schneider H, Russell SJ, Cox CJ, Bakker F, Henderson S, Rumsey F, Barrett J, Gibby M, Vogel JC (2004) Chloroplast phylogeny of asplenioid ferns based on $r b c L$ and $t r n L-F$ spacer sequences (Polypodiidae, Aspleniaceae) and its implications for biogeography. Systematic Botany, 29, 260274.

Shepherd LD, Perrie LR, Brownsey PJ (2008) Low copy nuclear DNA sequences reveal a predominance of allopolyploids in a New Zealand Asplenium fern complex. Molecular Phylogenetics and Evolution, 49, 240-248.

Tigerschiold E (1981) The Asplenium trichomanes complex in East Central Sweden. Nordic Journal of Botany, 1, 12-16.

van den Heede CG, Viane R, Chase MW (2003) Phylogenetic analysis of Asplenium subgenus Ceterach (Pteridophyta: Aspleniaceae) based on plastid and nuclear ribosomal ITS DNA sequences. American Journal of Botany, 90, 481-495.

Vogel JC, Russell SJ, Barrett SA, Gibby M (1996) A noncoding region of chloroplast DNA as a tool to investigate reticulate evolution in European Asplenium. In: Pteridology in Perspective (eds Camus JM, Johns RJ, Gibby M). Royal Botanic Garden, Kew, Richmond.

Wagner WH (1954) Reticulate evolution in the Appalachian Asplenium. Evolution, 8, 103-118.

Wang RX, Lu SG, Deng XC (2007) Cytotaxonomic studies of the Chinese pteridophytes: a review. Acta Phytotaxonomica Sinica, 45, 98-111. (in Chinese with English abstract) [王任 翔, 陆树刚, 邓晰朝 (2007) 中国偋类植物细胞分类学研 究概况. 植物分类学报, 45, 98-111.]

Wang ZR, Zhang F, Hou X (2003) A biosystematic study on Asplenium sarelii complex. Acta Botanica Sinica, 45, 1-14.

Werth CR, Guttman SI, Eshbaugh WH (1985) Recurring origins of allopolyploid species in Asplenium. Science, 228, 731-733.

Wood TE, Takebayashi N, Barker MS, Mayrose I, Greenspoon PB, Rieseberg LH (2009) The frequency of polyploid speciation in vascular plants. Proceedings of the National Academy of Sciences, USA, 106, 13875-13879.

Wu ZH (1999) Aspleniaceae. In: Flora Reipublicae Popularis Sinicae (ed. Editorial Committee of Flora Reipublicae Popularis Sinicae, Chinese Academy of Sciences), Tomus, 4(2). Science Press, Beijing. (in Chinese) [吴兆洪 (1999) 铁角偋科. 见: 中国植物志(中国科学院中国植物志编辑 委员会编), 4(2). 科学出版社, 北京.]

Yatabe Y, Masuyama S, Darnaedi D, Murakami N (2001) Molecular systematics of the Asplenium nidus complex from Mt. Halimun National Park, Indonesia: evidence for reproductive isolation among three sympatric $r b c L$ sequence types. American Journal of Botany, 88, 1517-1522.

Yatabe Y, Murakami N (2003) Recognition of cryptic species in the Asplenium nidus complex using molecular data-a progress report. Telopea, 10, 487-496.

Yatabe Y, Shonohara W, Matsumoto S, Murakami N (2009) Patterns of hybrid formation among cryptic species of bird-nest fern, Asplenium nidus complex (Aspleniaceae), in West Malesia. Botanical Journal of the Linnean Society, 160, 42-63.

Zhang XC (2012) Lycophytes and Ferns from China. Peking University Press, Beijing. (in Chinese) [张宪春 (2012) 中 国石松类和硕类植物. 北京大学出版社, 北京.]

Zhang XC, Wei R, Liu HM, He LJ, Wang L, Zhang G (2013) Phylogeny and classification of the extant lycophytes and ferns from China. Chinese Bulletin of Botany, 48, 119-137. (in Chinese with English abstract) [张宪春, 卫然, 刘红梅, 何丽娟, 王丽, 张钢 (2013) 中国现代石松类和硕类的系 统发育与分类系统. 植物学报, 48, 119-137.]

Zou XH, Ge S (2008) Conflicting gene trees and phylogenomics. Journal of Systematics and Evolution, 46, 795-807. (in Chinese with English abstract) [邹新慧, 葛颂 (2008) 基因 树冲突与系统发育基因组学研究. 植物分类学报, 46, 795-807.]

(责任编委: 严岳鸿 责任编辑: 间文杰)

\section{附录 Supplementary Material}

附录1 188种铁角硕科植物的染色体数目、细胞倍性和繁殖方式

Appendix 1 Chromosome number and ploidy levels of the 188 Aspleniaceae species http://www.biodiversity-science.net/fileup/PDF/2017117-1.pdf 
常艳芬. 铁角厥科的多倍化与物种多样性形成. 生物多样性, 2017, 25 (6): 621-626.

http://www.biodiversity-science.net/CN/10.17520/biods. 2017117

附录1 188种铁角烣科植物的染色体数目、细胞倍性和繁殖方式 $(\sqrt{ }$ 表示只有细胞倍性记录, 没有染色体数目 计数; $\mathrm{S}$ 表示有性生殖; $\mathrm{A}$ 表示无融合生殖)

Appendix 1 Chromosome number and ploidy levels of the 188 Aspleniaceae species. $\sqrt{ }$ Chromosome counts are not available but with ploidy level recorded; S, sexual reproduction; A, apomictic reproduction.

\begin{tabular}{|c|c|c|c|c|c|c|c|c|c|}
\hline 种名 Species & $\mathrm{S} / \mathrm{A}$ & $2 X$ & $3 X$ & $4 X$ & $6 \mathrm{X}$ & $8 \mathrm{X}$ & $10 \mathrm{X}$ & $12 \mathrm{X}$ & $16 \mathrm{X}$ \\
\hline Asplenium abscissum & & 72 & & & & & & & \\
\hline 黑色铁角蕨 A. adiantum-nigrum & & & & $\sqrt{ }$ & $\sqrt{ }$ & & & & \\
\hline 合生铁角蕨 A. adnatum & & & & $\sqrt{ }$ & & & & & \\
\hline A. adulterinum & & & & 144 & & & & & \\
\hline A. aethiopicum & & & & 144 & & 288 & & $\sqrt{ }$ & $\sqrt{ }$ \\
\hline 匙形铁角硕 A. affine & & & & & & $\sqrt{ }$ & & & \\
\hline 西部铁角葓 A. aitchisonii & & & & $\sqrt{ }$ & $\sqrt{ }$ & & & & \\
\hline 阿尔泰铁角蕨 A. altajense & & & & $\sqrt{ }$ & & & & & \\
\hline A. alternifolium & & & 108 & 144 & & & & & \\
\hline A. anceps & $\mathrm{S}$ & 72 & & 144 & & & & & \\
\hline 广布铁角蕨 A. anogrammoides & & & & $\sqrt{ }$ & & & & & \\
\hline 大鳞巢蕨 A. antiquum & & & & $\sqrt{ }$ & & & & & \\
\hline 狭翅巢蕨 A. antrophyoides & & & & $\sqrt{ }$ & & & & & \\
\hline 黑鳞铁角蕨 A. asterolepis & & & & $\sqrt{ }$ & & & & & \\
\hline A. aureum & A & & & 144 & 216 & 288 & & & \\
\hline A. auritum & A & 72 & & 144 & & & & & \\
\hline A. australasicum & & & & 144 & & & & & \\
\hline 华南铁角硕 A. austrochinense & & & & $\sqrt{ }$ & & $\sqrt{ }$ & & & \\
\hline A. azomanes & & & & 144 & & & & & \\
\hline A. azoricum & & & & 144 & & & & & \\
\hline A. balearicum & & 72 & & & & & & & \\
\hline A. belangeri & & & & 144 & & & & & \\
\hline A. billotii & & & & 144 & & & & & \\
\hline A. bradleyi & & & & $\sqrt{ }$ & & & & & \\
\hline A. bulbiferum & & & & 144 & & & & & \\
\hline 大盖铁角蕨 A. bullatum & & & & $\sqrt{ }$ & & $\sqrt{ }$ & & & \\
\hline A. capense & & 72 & & & & & & & \\
\hline 线柄铁角偋 A. capillipes & $\mathrm{S}$ & $\sqrt{ }$ & & $\sqrt{ }$ & & & & & \\
\hline 东海铁角蕨 A. castaneoviride & & & & 144 & & & & & \\
\hline 高加索铁角蕨 A. caucasicum & $\mathrm{S}$ & 72 & & $\sqrt{ }$ & & & & & \\
\hline A. caudatum & & & & 144 & & & & & \\
\hline A. centrafricanum & & & & 140 & & & & & \\
\hline 药蕨 A. ceterach & & $\sqrt{ }$ & $\sqrt{ }$ & $\sqrt{ }$ & $\sqrt{ }$ & & & & \\
\hline A. chathamense & & & & 144 & & & & & \\
\hline A. chihuahuense & & & & & 216 & & & & \\
\hline A. cimmeriorum & & & & & & 288 & & & \\
\hline A. claussenii & & 72 & & & & & & & \\
\hline A. cordatum & & 72 & & 144 & & & & & \\
\hline 线裂铁角硕 A. coenobiale & & & & $\sqrt{ }$ & & & & & \\
\hline 壮乡铁角蒴 A. cornutissimum & & & & $\sqrt{ }$ & & & & & \\
\hline 毛轴铁角蒴 A. crinicaule & & & & $\sqrt{ }$ & & $\sqrt{ }$ & & $\sqrt{ }$ & \\
\hline A. cristatum & $\mathrm{S}$ & 72 & & 144 & & & & & \\
\hline 乌来铁角蕨 A. cuneatiforme & & & & $\sqrt{ }$ & & & & & \\
\hline A. curtissii & A & & 108 & & & & & & \\
\hline A. cyprium & & & & & 216 & & & & \\
\hline 苍山築 A. dalhousiae & $\mathrm{S}$ & $\sqrt{ }$ & & $\sqrt{ }$ & & & & & \\
\hline A. decrescens & & & & 144 & & & & & \\
\hline 水鳖硕 A. delavayi & & & & $\sqrt{ }$ & & & & & \\
\hline A. dentatum & A & 72 & 108 & & & & & & \\
\hline A. diplazisorum & & & & 144 & & & & & \\
\hline 圆叶铁角蕨 A. dolomiticum & $\mathrm{S}$ & 72 & & 144 & & & & & \\
\hline A. dolosum & & & & & 216 & & & & \\
\hline
\end{tabular}


常艳芬. 铁角蒝科的多倍化与物种多样性形成. 生物多样性, 2017, 25 (6): 621-626. http://www.biodiversity-science.net/CN/10.17520/biods. 2017117

\begin{tabular}{|c|c|c|c|c|c|c|c|c|c|}
\hline 种名 Species & S/A & $2 \mathrm{X}$ & $3 \mathrm{X}$ & $4 \mathrm{X}$ & $6 \mathrm{X}$ & $8 \mathrm{X}$ & $10 \mathrm{X}$ & $12 \mathrm{X}$ & $16 \mathrm{X}$ \\
\hline A. ebenoides & & 72 & & 144 & & & & & \\
\hline 剑叶铁角蕨 A. ensiforme & & & & $\sqrt{ }$ & & & & & \\
\hline A. erectum & & 72 & & & & & & & \\
\hline 云南铁角蒝 A. exiguum & & & & $\sqrt{ }$ & & & & & \\
\hline 网脉铁角蕨 A. finlaysonianum & & & & $\sqrt{ }$ & & $\sqrt{ }$ & & & \\
\hline A. fissum & & 72 & & & & & & & \\
\hline A. flabellifolium & & & & $\sqrt{ }$ & $\sqrt{ }$ & & & $\sqrt{ }$ & \\
\hline A. flaccidum & & & & 144 & & & & & \\
\hline 西藏铁角蕨 A. fontanum & S & $\sqrt{ }$ & & $\sqrt{ }$ & & & & & \\
\hline A. foresiense & S & & & 144 & & & & & \\
\hline 南海铁角濒 A. formosae & & & & $\sqrt{ }$ & & & & & \\
\hline 易变铁角硕 A. fugax & & & & $\sqrt{ }$ & & & & & \\
\hline 腺齿铁角䔊 A. glanduliserrulatum & & & & $\sqrt{ }$ & & & & & \\
\hline A. goudeyi & & & & $\sqrt{ }$ & & & & & \\
\hline A. grevillei & & 72 & & & & & & & \\
\hline 厚叶铁角蕨 A. griffithianum & S & 72 & & 144 & & & & $\sqrt{ }$ & \\
\hline 撕裂铁角蕨 A. gueinzianum & & & & $\sqrt{ }$ & & & & & \\
\hline 海南铁角蕨 A. hainanense & & & $\sqrt{ }$ & $\sqrt{ }$ & & & & & \\
\hline A. haughtonii & & & & $\sqrt{ }$ & & & & & \\
\hline A. haussknechtii & & & & 144 & & & & & \\
\hline A. helii & S & 72 & & & & & & & \\
\hline A. hemionitis & & 72 & & & & & & & \\
\hline A. heterochroum & & & & 144 & 216 & & & & \\
\hline A. hindusthanensis & & 72 & & & & & & & \\
\hline A. hobdyi & & & & $\sqrt{ }$ & & & & & \\
\hline 江南铁角硕 A. holosorum & & & & $\sqrt{ }$ & $\sqrt{ }$ & & & & \\
\hline A. hostmannii & & & 108 & 144 & & & & & \\
\hline 扁柄巢蕨 A. humbertii & & & & & $\sqrt{ }$ & & & & \\
\hline A. hybridum & & 72 & & & & & & & \\
\hline A. inaequilaterale & S & 72 & & 144 & & & & & \\
\hline 虎尾铁角蕨 A. incisum & S & $\sqrt{ }$ & & $\sqrt{ }$ & & & & & \\
\hline 胎生铁角蕨 A. indicum & S & $\sqrt{ }$ & & $\sqrt{ }$ & & $\sqrt{ }$ & & & \\
\hline A. indopakistanicum & & 72 & & & & & & & \\
\hline 贵阳铁角蕨 A. interjectum & S & $\sqrt{ }$ & & $\sqrt{ }$ & & & & & \\
\hline A. juglandifolium & & & & 144 & & & & & \\
\hline 对开蕨 A. komarovii & & $\sqrt{ }$ & & $\sqrt{ }$ & & & & & \\
\hline 西疆铁角蕨 A. kukkonenii & & & & $\sqrt{ }$ & & & & & \\
\hline A. lepidum & & & & 144 & & & & & \\
\hline A. loxoscaphoides & & & & 140 & & & & & \\
\hline 沪山铁角蕨 A. lushanense & A & $\sqrt{ }$ & & $\sqrt{ }$ & & & & & \\
\hline A. macilentum & & & & & & 288 & & & $\sqrt{ }$ \\
\hline 大叶苍山蕨 A. magnificum & & & & & & $\sqrt{ }$ & & & \\
\hline A. majoricum & & & & 144 & & & & & \\
\hline A. marinum & & 72 & & & & & & & \\
\hline A. milnei & & & & 144 & & & & & \\
\hline A. monanthes & $\mathrm{A}$ & & 108 & & 216 & & & & \\
\hline A. montanum & & 72 & & & & & & & \\
\hline A. morganii & & & 108 & & & & & & \\
\hline A. murbeckii & S & & & 144 & & & & & \\
\hline 巢硕 A. nidus & & & & $\sqrt{ }$ & $\sqrt{ }$ & & & & \\
\hline 倒挂铁角蕨 A. normale & S & $\sqrt{ }$ & & $\sqrt{ }$ & $\sqrt{ }$ & $\sqrt{ }$ & & & \\
\hline 黑鳞巢蕨 A. oblanceolatum & & & & $\sqrt{ }$ & & & & & \\
\hline A. obovatum & S & 72 & & 144 & & & & & \\
\hline A. onopteris & S & 72 & & 144 & & & & & \\
\hline A. pagesii & S & & & & 216 & & & & \\
\hline A. paleaceum & & & & 144 & & & & & \\
\hline
\end{tabular}




\begin{tabular}{|c|c|c|c|c|c|c|c|c|c|}
\hline 种名 Species & S/A & $2 \mathrm{X}$ & $3 X$ & $4 \mathrm{X}$ & $6 \mathrm{X}$ & $8 X$ & $10 \mathrm{X}$ & $12 \mathrm{X}$ & $16 \mathrm{X}$ \\
\hline 疏脉苍山蕨 A. paucivenosum & & & & $\sqrt{ }$ & & & & & \\
\hline 北京铁角硕 A. pekinense & & & & $\sqrt{ }$ & & & & & \\
\hline A. petersenii & & & & 144 & & & & & \\
\hline A. petrarchae & $\mathrm{S}$ & 72 & 108 & 144 & & & & & \\
\hline A. phillipsianum & & 72 & & & & & & & \\
\hline 长叶巢蕨 A. phyllitidis & & & & $\sqrt{ }$ & & & & & \\
\hline A. pinnatifidum & & & & 144 & & & & & \\
\hline A. platyneuron & & 72 & & & & & & & \\
\hline A. plenum & & & & $\sqrt{ }$ & & & & & \\
\hline 镰叶铁角硕 A.polyodon & & & & & $\sqrt{ }$ & & $\sqrt{ }$ & & \\
\hline 长叶铁角䔊 A. prolongatum & & & & $\sqrt{ }$ & & & & & \\
\hline A. protomajoricum & & & & 144 & & & & & \\
\hline 假大羽铁角䔊 A. pseudolaserpitiifolium & & & & & & $\sqrt{ }$ & & & \\
\hline 斜裂铁角蕨 A. pseudopraemorsum & & & & $\sqrt{ }$ & & & & & \\
\hline 叶基宽铁角硕 A. pulcherrimum & & & & $\sqrt{ }$ & & & & & \\
\hline A. pumilum & & 72 & & & & & & & \\
\hline A. punjabense & & & & & 216 & & & & \\
\hline A. radicans & & 72 & & & & 288 & & & \\
\hline A. recoderi & & & 108 & & 216 & & & & \\
\hline A. reichsteinii & & 72 & 108 & & & & & & \\
\hline A. resiliens & & & & & 216 & & & & \\
\hline A. rhizophyllum & & $\sqrt{ }$ & & & & & & & \\
\hline 骨碎补铁角蒴 A. ritoense & & & & $\sqrt{ }$ & & & & & \\
\hline 瑞丽铁角蕨 A.rockii & & & & & $\sqrt{ }$ & & & & \\
\hline A. rosselloi & & 72 & & & & 288 & & & \\
\hline A. rouyi & & & & 144 & & & & & \\
\hline 过山蕨 A. ruprechtii & S & $\sqrt{ }$ & & $\sqrt{ }$ & & & & & \\
\hline 卵叶铁角硕 A. ruta-muraria & & & & $\sqrt{ }$ & & & & & \\
\hline A. rutifolium & & & & 140 & & 280 & & & \\
\hline A. salicifolium & & & & & & 288 & & & \\
\hline A. samarkandense & & & & 144 & & & & & \\
\hline 岭南铁角硕 A. sampsonii & & & & & & & $\sqrt{ }$ & & \\
\hline 华中铁角硕 A. sarelii & S & 72 & & 144 & & & & & \\
\hline A. scolopendrium & & $\sqrt{ }$ & & & & & & & \\
\hline 狭叶铁角蕨 A. scortechinii & & & & $\sqrt{ }$ & $\sqrt{ }$ & & & $\sqrt{ }$ & \\
\hline 近变异铁角葓 A. semivarians & S & $\sqrt{ }$ & & $\sqrt{ }$ & & & & & \\
\hline 叉叶铁角蒴 A. septentrionale & S & $\sqrt{ }$ & & $\sqrt{ }$ & & & & & \\
\hline A. serra & & & & & & 288 & & & \\
\hline A. serratum & & & & 144 & & 288 & & & \\
\hline A. sertularioides & & & & 140 & & & & & \\
\hline A. simplicifrons & & & & 144 & & & & & \\
\hline A. sleepiae & & 72 & & & & 288 & & & \\
\hline A. souchei & & & & 144 & & 288 & & & \\
\hline A. subhastatum & & & & & & 288 & & & \\
\hline A. surrogatum & & 72 & & & & & & & \\
\hline A. tabulense & & & & $\sqrt{ }$ & & & & & \\
\hline 膜连铁角蕨 A. tenerum & & & & $\sqrt{ }$ & & & & & \\
\hline 细茎铁角蕨 A. tenuicaule & S & $\sqrt{ }$ & & $\sqrt{ }$ & & & & & \\
\hline 细裂铁角硕 A. tenuifolium & S & 72 & & 144 & & & & & \\
\hline A. theciferum & & & & 140 & & 280 & & $\sqrt{ }$ & \\
\hline A. ticinense & & 72 & 108 & & & & & & \\
\hline 铁角蕨 A. trichomanes & S & $\sqrt{ }$ & $\sqrt{ }$ & $\sqrt{ }$ & $\sqrt{ }$ & & & & \\
\hline 台南铁角硕 A. trigonopterum & & & & & & $\sqrt{ }$ & & & \\
\hline 三翅铁角硕 A. tripteropus & S & $\sqrt{ }$ & & $\sqrt{ }$ & & & & & \\
\hline A. triquetrum & & & & 144 & & & & & \\
\hline A. troodeum & & & & & $\sqrt{ }$ & & & & \\
\hline
\end{tabular}


常艳芬. 铁角蒝科的多倍化与物种多样性形成. 生物多样性, 2017, 25 (6): 621-626. http://www.biodiversity-science.net/CN/10.17520/biods. 2017117

\begin{tabular}{|c|c|c|c|c|c|c|c|c|c|}
\hline 种名 Species & S/A & $2 \mathrm{X}$ & $3 \mathrm{X}$ & $4 X$ & $6 \mathrm{X}$ & $8 \mathrm{X}$ & $10 \mathrm{X}$ & $12 \mathrm{X}$ & $16 \mathrm{X}$ \\
\hline A. trudellii & & & 108 & & & & & & \\
\hline A. ulbrichtii & & & & 144 & & & & & \\
\hline 变异铁角蕨 A. varians & $\mathrm{S}$ & $\sqrt{ }$ & & $\sqrt{ }$ & & & & & \\
\hline 欧亚铁角蕨 A. viride & $\mathrm{S}$ & $\sqrt{ }$ & & $\sqrt{ }$ & $\sqrt{ }$ & & & & \\
\hline 狭翅铁角蕨 A. wrightii & & & & & & $\sqrt{ }$ & $\sqrt{ }$ & $\sqrt{ }$ & \\
\hline A. wudangense & & & & 144 & & & & & \\
\hline A. $\times$ artanense & & 72 & & 144 & & & & & \\
\hline A. $\times$ centovallense & & & & 144 & & & & & \\
\hline A. $\times$ diasii & & & & & 216 & & & & \\
\hline A. $\times$ sarniense & & 72 & & 144 & 216 & & & & \\
\hline A. $\times$ sollerense & & & & 144 & & & & & \\
\hline A. $\times$ tyrrhenicum & & 72 & & & & & & & \\
\hline 棕鳞铁角蕨 A. yoshinagae & & & & & & $\sqrt{ }$ & & & \\
\hline 无配膜叶铁角蕨 H. apogamum & $\mathrm{A} / \mathrm{S}$ & & 117 & 156 & & & & & \\
\hline 细辛膜叶铁角蒝 H. cardiophyllum & S & $\sqrt{ }$ & & $\sqrt{ }$ & & & & & \\
\hline 齿果膜叶铁角蕨 H. cheilosorum & $\mathrm{S}$ & $\sqrt{ }$ & $\sqrt{ }$ & $\sqrt{ }$ & & & & & \\
\hline 切边膜叶铁角蕨 H. excisum & $\mathrm{S}$ & $\sqrt{ }$ & & $\sqrt{ }$ & & & & & \\
\hline 东亚膜叶铁角偋 H. hondoense & $\mathrm{A} / \mathrm{S}$ & & $\sqrt{ }$ & $\sqrt{ }$ & & & & & \\
\hline 单边膜叶铁角蕨 H. murakami-hatanakae & & $\sqrt{ }$ & & & & & & & \\
\hline 荫湿膜叶铁角蕨 H. obliquissimum & $\mathrm{A} / \mathrm{S}$ & $\sqrt{ }$ & $\sqrt{ }$ & $\sqrt{ }$ & & & & & \\
\hline 绿杆膜叶铁角蕨 H. obscurum & & $\sqrt{ }$ & & $\sqrt{ }$ & & & & & \\
\hline 尖峰岭膜叶铁角蕨 H. pseudobscurum & & $\sqrt{ }$ & & $\sqrt{ }$ & & & & & \\
\hline 小膜叶铁角蕨 H. subnormale & & 76 & & 152 & & & & & \\
\hline H. unilaterale & & $\sqrt{ }$ & $\sqrt{ }$ & $\sqrt{ }$ & & & & & \\
\hline
\end{tabular}

